\title{
ANAESTHETIC MANAGEMENT OF THE PATIENT WITH RHEUMATOID ARTHRITIS
}

\author{
LeonaRd C. Jenkins, B.A., M.D., C.M., F.R.C.P.(c), AND \\ Robert W. McGraw, M.D., F.r.c.s. (c) ${ }^{\circ}$
}

Rheumatom aRTHRitis is a relatively common disease. Several surveys made in temperate climates indicate that the incidence of rheumatoid arthritis among those over 15 years of age is approximately 3 per cent. ${ }^{1}$ Women are more often affected than men. A large proportion of patients become incapacitated. In one study, 58 per cent of patients managed conservatively were unable to carry out their ordinary occupation or duties. ${ }^{1}$

Although many patients with rheumatoid arthritis may present for surgery unrelated to their disease, an increasing number now present for definitive corrective surgery. The operative sites, indications for surgery, and common operative procedures performed for these patients by the Department of Orthopaedic Surgery at the Vancouver General Hospital are summarized in Table I. It is important, and not always appreciated, that anaesthesiologists and surgeons should have due regard for the hazards associated with the management of the patient with rheumatoid arthritis. ${ }^{2,3}$ The hazards which should be anticipated in the approach to management of this type of patient are summarized in Table II. The anaesthetic implications of these hazards will be discussed in relationship to the preoperative, operative, and postoperative periods.

\section{Preoperattve Period}

Careful assessment and management of rheumatoid arthritic patients is important in the preoperative period, and sets the stage for management during operation and in the postoperative period.

\section{Respiratory status}

Airway. During the preanaesthetic visit it is valuable to review the respiratory skeletal profile of the rheumatoid arthritic patient, because rheumatoid deformities may predispose to airway obstruction when the patient is unconscious during anaesthesia. The clinical presence of hypoplastic mandible, temporo-mandibular ankylosis, reduced cervical spine motions, atlanto-axial subluxation, or low cervical subluxation necessitate verification and clarification of the degree of involvement by preoperative skeletal radiology. Patients who present with hoarseness, dyspnoea on exertion, stridor, dysphagia, or throat fulness when speaking should be suspected of having a stenotic-rheumatoid arthritis involvement of the crico-arytenoid cartilages with narrowing of the laryngeal opening. There may also be an associated rheuma-

'Divisions of Anaesthesia and Orthopaedic Surgery, Vancouver General Hospital and University of British Columbia. 
TABLE I

Corrective Surgery for Rheumatoid Arthritis

\begin{tabular}{|c|c|}
\hline Operative site & Common operative procedure \\
\hline Neck & atlanto-axial arthrodesis \\
\hline Shoulder & $\begin{array}{l}\text { synovectomy and partial excision } \\
\text { acromion }\end{array}$ \\
\hline Elbow & $\begin{array}{l}\text { synovectomy and radial head } \\
\text { excision; resection arthroplasty }\end{array}$ \\
\hline Wrist & $\begin{array}{l}\text { synovectomy and excision distal } \\
\text { ulna }\end{array}$ \\
\hline Hand & $\begin{array}{l}\text { metacarpal phalangeal arthroplasty } \\
\text { and fiexor and extensor } \\
\text { tenosynovectomy }\end{array}$ \\
\hline Hip & $\begin{array}{l}\text { cup or total replacements } \\
\text { arthroplasty }\end{array}$ \\
\hline Knee & $\begin{array}{l}\text { synovectomy (of ten bilateral) } \\
\text { arthroplasty }\end{array}$ \\
\hline Foot & $\begin{array}{l}\text { resection arthroplasty (often } \\
\text { bilateral }\end{array}$ \\
\hline
\end{tabular}

TABLE II

Anaesthetic Hazards in Patients with Rheumatoid Arthritis

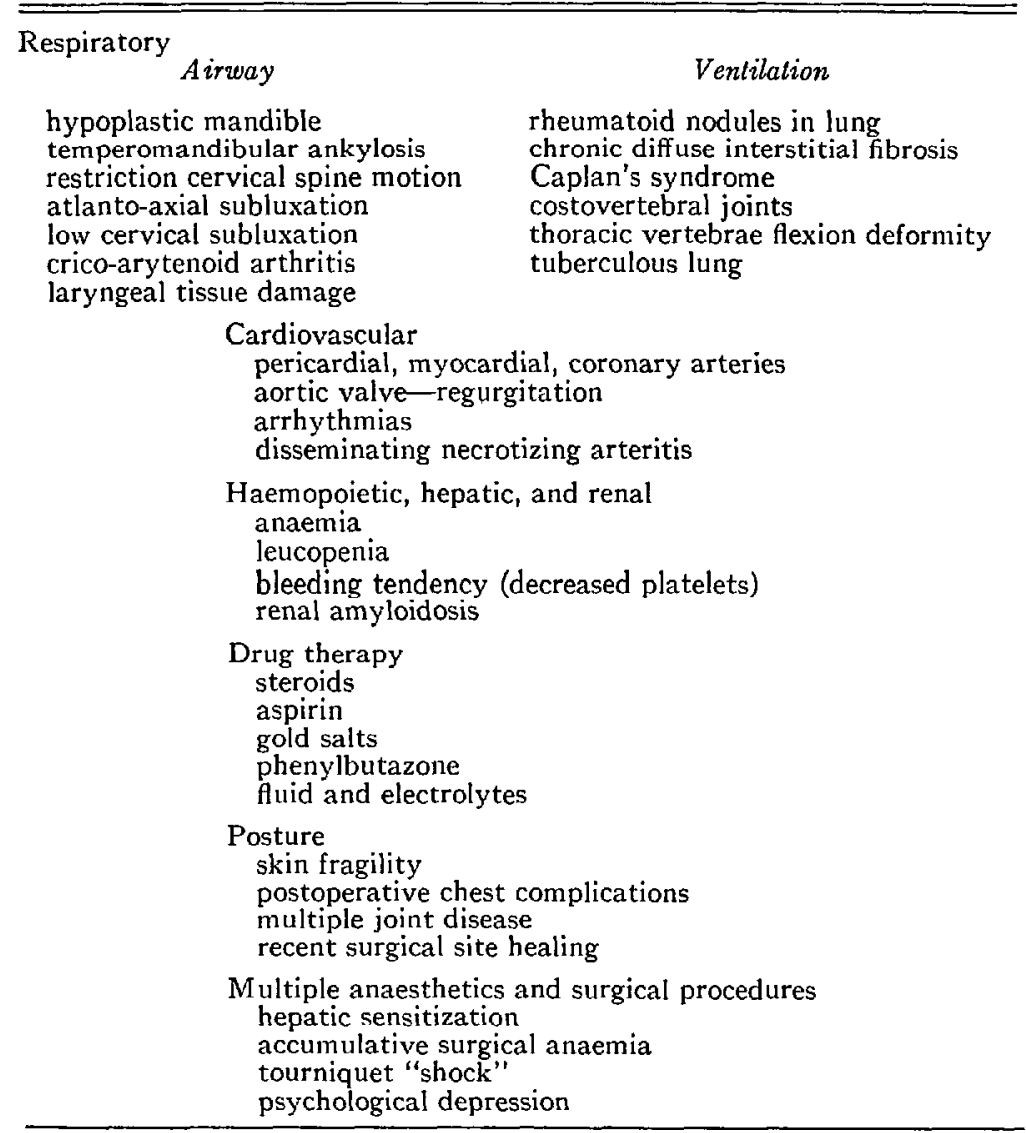


toid destruction of laryngeal tissues. Rheumatoid arthritis of the crico-arytenoid joint is fairly common. Bienenstock and associates ${ }^{4}$ reported a 26 per cent incidence of crico-arytenoid arthritis in their series. A diagnosis is made by preoperative indirect laryngoscopy. This may show redness and oedema of the tissue around the joint. There is decreased crico-arytenoid motion or bowing of the vocal cords during inspiration. Vocal cord movement during respiration is diminished when there is chronic joint fixation. If general anaesthesia is contemplated, a preoperative tracheostomy is advisable in the patient with rheumatoid arthritis of the cricoarytenoid joint.

Ventilation. Patients with rheumatoid arthritis are known to have specific pulmonary lesions. The occurrence of lung lesions varies considerably. The lesions that have been described are rheumatoid nodules, ${ }^{5}$ cysts with honeycombing of the lung, diffuse interstitial pulmonary fibrosis, and non-specific fibrosis. A particular type of rheumatoid lung involvement in combination with pneumoconiosis of coal miners is known as Caplan's syndrome. ${ }^{6}$ In British Columbia, an unrecognized active tuberculous lung is not uncommon in Indian patients with rheumatoid arthritis, particularly when these patients have been on long-term steroid therapy. Thus, a preoperative chest $X$-ray is essential. Chronic diffuse interstitial pulmonary fibrosis is the most common pulmonary lesion. ${ }^{7}$ Dyspnoea with an associated cough, cyanosis at rest, clubbing of fingers, hyperventilation, and crepitations throughout the lung fields on auscultation are signs and symptoms requiring attention. Pulmonary function tests may show decreased lung compliance, and diminished diffusing capacity with low $\mathrm{PCO}_{2}$ and arterial desaturation. Costochondral involvement and flexion deformity of the thoracic vertebrae, particularly seen in the ankylosing spondylitis type of rheumatoic disease, grossly limit chest wall expansion. Patients with these conditions should have preoperative blood gas analyses $\left(\mathrm{PCO}_{2}, \mathrm{Po}_{2}\right.$, and $\left.\mathrm{pH}\right)$. Baseline tidal and minute volumes should be recorded (Wright respirometer). These preoperative values will serve as guides for management of ventilation during the operative and postoperative periods.

\section{Cardiovascular status}

Involvement of the heart is not uncommon in rheumatoid arthritis. The pericardium, myocardium, and endocardium may be severely involved. There may be damage to coronary vessels. A variety of electrocardiographic and clinical cardiac abnormalities may be apparent. ${ }^{8}$ These may present as heart murmurs of all types, as enlargement of the heart, as arrhythmias secondary to defects of myocardial conduction, and as signs and symptoms of pericardial disease. The frequency of these manifestations is such that even in younger patients a complete cardiac evaluation is recommended. As a minimum, this entails a preoperative ECG and cardiothoracic ratio estimation from the chest film.

It is important to realize that patients with ankylosing spondylitis have an appreciable incidence of valvular disease, particularly aortic regurgitation. ${ }^{\theta}$ This is due to thickening and shortening of the valve cusps with widening of the annulus fibrosis and stretching of the attachments of the annulus and cusps to the aortic wall, allowing the valve to sag downward. Since regurgitant aortic flow 
places strain on the left ventricle which is compensated by increased stroke volume, ${ }^{10}$ if hypotension is allowed during anaesthesia in these cases it may cause left ventricular decompensation with acute heart failure and its consequences. Maintenance of a stable haemodynamic pattern is essential in this type of patient during all periods of the surgical-anaesthetic sequence.

Patients with long-standing rheumatoid arthritis may develop disseminated necrotizing arteritis. This may lead secondarily to generalized polyneuropathy due to poor nourishment of the peripheral nerves. ${ }^{11}$ A similar necrotizing arteritis has been noted in large and small bowel at necroscopy and is associated clinically with marked diarrhoea with attendant fever and electrolyte imbalance. ${ }^{12}$

Haemopoietic, hepatic, and renal function

Haemopoietic function. Patients with rheumatoid arthritis are frequently anaemic. A determination of preoperative haemoglobin is therefore mandatory. Although the anaemia is of the hypochromic microcytic variety it is usually refractory to all forms of therapy except blood transfusion. Even intravenous iron has only a slightly improving effect. Blood transfusion improves haemoglobin concentration. The preoperative haemoglobin should be brought to at least 10.0 gm for elective surgery. Packed red blood cell transfusions should be administered if, as is often the case, the body weight is small, or if a potential cardiac failure problem exists. Causes contributing to the anaemia may be a bleeding peptic ulcer ( secondary to long-term steroid therapy), or bleeding tendency secondary to chronic acetylsalicylic acid, phenylbutazolidine, or gold salt therapy with associated leucopenia and decrease in platelet count. Stools should be examined for occult blood in the preoperative period. If the haemoglobin is not checked prior to each surgical procedure anaemia may develop as a result of accumulative surgical blood loss due to the multiple surgical procedures performed. The presence of anaemia signifies much more than absolute deficiency in haemoglobin. It may mean that there is also hypovolaemia and hypoproteinaemia. It indicates a definite decrease in oxygen carrying capacity and oxygen availability. ${ }^{13}$ Anaemic patients withstand slight degrees of hypoxia and blood loss poorly. Their handling of anaesthetic drugs may be significantly altered. Plasma protein alterations can adversely affect protein binding and redistribution of drugs. ${ }^{14,10}$

Renal function. As measured by decreased creatinine clearance testing, patients with rheumatoid arthritis are three times more likely to have impairment of renal function, secondary to amyloidosis, than controls. ${ }^{15}$ Decreased renal function dictates the need for the omission of anaesthetic drugs that are primarily excreted by the kidneys, in all phases of anaesthetic management. This aspect is discussed specifically later. Deaths associated with anaesthesia in rheumatoid arthritic patients with renal amyloidosis have been attributed to impaired renal function; ${ }^{3}$ yet it is suggested by one report ${ }^{16}$ that severe amyloidosis, proven by renal biopsy, may cause only very slight renal dysfunction.

Hepatic function. Liver function is not seriously affected in rheumatoid arthritic patients. ${ }^{17}$ Possible hepatic sensitization to halogenated hydrocarbon anaesthetics must be considered when multiple surgical procedures are undertaken at short intervals. ${ }^{18.19}$ 


\section{Drug therapy}

The anaesthetic implications of drug therapy and their management are discussed in relation to preanaesthetic medication. Rheumatoid arthritic patients may be on long-term steroid therapy. The effects of chronic acetylsalicylic acid, gold salts, and phenylbutazone therapy are discussed in relation to haemopoietic, liver, and renal function.

The features of the comprehensive assessment of these patients required to evaluate the operative risk and to determine the hazards attending their management are reiterated in summary form in Table III.

TABLE III

Preoperative Assessment of Medical Status for the Rheumatoid Arthritic Patient

\begin{tabular}{ll}
\hline \hline Skeletal radiology & Haemoglobin \\
Indirect laryngoscopy & White blood cell, platelet count \\
Chest X-ray & Occult blood stools \\
Pulmonary function tests & Urinalysis \\
Blood gas analysis & Creatinine clearance \\
ECG & Drug therapy history \\
Cardio-thoracic ratio & Previous recent general anaesthetics \\
\hline
\end{tabular}

\section{Preanaesthetic medication}

Patients with rheumatoid arthritis may receive large doses of steroids for lengthy periods. This will lead to inhibition of the adrenal cortex and in some patients to inhibition of the anterior pituitary or the hypothalamic nuclei controlling the anterior pituitary. The endogenous output of corticosteroids by the adrenal cortex or the therapeutic dose of steroids which the patient is receiving is likely to be inadequate for the added stress of the preoperative, operative, and postoperative periods. A severe and prolonged hypotension, refractory to all therapy save additional steroids, will develop. Therefore, supplemental steroids must be given when the patient is currently on corticosteroids, or has been on prolonged therapy within one year of surgery, or on prolonged therapy up to two years prior to surgery when evidence of hypercortisonism existed. A supplemental steroid schedule is: $200 \mathrm{mg}$ cortisone acetate on the evening prior to surgery; $200 \mathrm{mg}$ cortisone acetate intramuscularly the morning of surgery; $100 \mathrm{mg}$ hydrocortisone intravenously during surgery. During the postoperative period the patient is weaned step-wise from the steroid therapy to normal dosage. Opiates (morphine, meperidine, or omnopon), phenothiazines (promethazine), barbiturates, and belladonna drugs (atropine, scopolamine), may be given at premedication to patients with rheumatoid arthritis. Of the barbiturates phenobarbital is contraindicated by renal amyloidosis. The guiding principle here is moderation in dosage because of the often obscured possible presence of renal impairment and decreased cardiac and respiratory reserve.

\section{Induction of anaesthesia}

General anaesthesia versus regional block. Because of the respiratory hazards associated with general anaesthesia, thought should always be given to the possibility of using a regional anaesthetic procedure. For pelvic and lower limb sur- 
gery, spinal or peridural anaesthesia is applicable. This approach may be impossible because of anatomical deformity and calcification of the lumbar ligaments. Regional anaesthesia is contraindicated if nerves are involved by the rheumatoid process and paraesthesia or neurologic deficit has resulted. This may occur from pressure on the nerve by direct invasion of rheumatoid nodules leading to a neuritis. In addition, patients with long-standing disease may develop disseminated necrotizing arteritis. This may result in generalized polyneuropathy, and is believed to be due to poor nourishment of the nerves. Local anaesthetic injection may exacerbate this neuropathy. ${ }^{11}$ The trend is toward general anaesthesia, since these patients have multiple joint involvement and several regions of the body may have corrective surgical procedures performed simultaneously by a team of orthopaedic surgeons. This approach reduces the hazards of multiple anaesthetics and improves the psychological status of the patient. It is difficult to provide adequate relaxation and analgesia for all surgical sites simultaneously by regional block techniques.

The airway. Problems of the airway should be anticipated in the respiratory assessment of the patient. A sleep dose of thiopental and a short-acting muscle relaxant (succinylcholine) may be given only after a great deal of forethought with regard to the ability to maintain an airway once the patient is unconscious and assisted or controlled ventilation is necessary. In the presence of a hypoplastic mandible, fixation of the temporomandibular joints or marked restriction of movement of the cervical vertebrae, even opening of the mouth, may be very difficult and visualization of the larynx for oral endotracheal intubation impossible. It is thus frequently necessary to intubate the patient awake, following local anaesthetic to the oropharynx. Blind nasotracheal intubation is frequently the method of choice. A tracheostomy under local anaesthesia should be done before induction of general anaesthesia when involvement of crico-arytenoid, and/or laryngeal tissue are severe. It is important to realize that vertebral erosion and subluxation may occur at the atlantoaxial joint, or at lower cervical sites. This may develop even spontaneously. ${ }^{20}$ These defects are more prevalent than often is recognized. Therefore, the head and neck should always be manipulated with the greatest of care during endotracheal intubation and positioning of the patient. Fatal spinal cord compression secondary to atlantoaxial subluxation has been described in a patient with rheumatoid arthritis who died a few hours after iridectomy. ${ }^{20} \mathrm{~A}$ transient basilar artery syndrome has been observed in one of our rhcumatoid arthritic patients, associated with spontaneous subluxation of the atlantoaxial joint prior to anaesthesia. ${ }^{12}$

\section{Maintenance anaesthesia}

\section{Operative Period}

Because ventilatory reserve may be greatly diminished in the patient with rheumatoid arthritis, assisted or controlled ventilation is necessary during general anaesthesia. Physiological levels of ventilation may be maintained by periodic monitoring of arterial blood gases for $\mathrm{Po}_{2}, \mathrm{PCO}_{2}$, and $\mathrm{pH}$, and of tidal volumes (VT) and minute volumes (VM) (Wright respirometer).

Cardiac reserve may be decreased. General and regional anaesthetics are ad- 
ministered in such a way that hypotension is avoided. This is particularly important when there is associated aortic regurgitation, since these patients have a relatively fixed cardiac output and acute coronary insufficiency may be precipitated by a hypotensive episode. Attention is given to early and adequate replacement of blood loss, as these patients often have anaemia.

A variety of general anaesthetic agents have been used in maintenance of anaesthesia for rheumatoid arthritic patients at the Vancouver General Hospital. Provided that attention is given to the established pharmacological effects of any given agent, many approaches are feasible. Combinations that have successfully been employed are: nitrous-oxide-oxygen and halothane, or methoxyflurane; nitrous-oxide-oxygen and gallamine or d-tubocurare with hyperventilation; nitrous-oxide-oxygen and meperidine or alphaprodine or Innovar (fentanyl and droperidol); cyclopropane and oxygen.

Low concentrations of halothane are used. Methoxyflurane should perhaps be avoided when there is significant renal amyloidosis. ${ }^{15,16}$ The use of both halothane and methoxyflurane may be questioned when multiple anaesthetics are to be given within a short period because of the possibility of hepatic sensitization (this last problem is not yet clarified, and this precaution may not be warranted). ${ }^{18,19}$

Gallamine is to be avoided when renal function is impaired by amyloidosis, and the use of the surgical cautery limits the use of cyclopropane.

\section{Posturing}

Great care must be taken in posturing the patient with rheumatoid arthritis. The skin is fragile due to weight loss, steroid therapy, and the nature of the disease; pressure sores or damage to nerves and occlusion of circulation to a limb may result from inappropriate postures; joint involvement may greatly restrict positioning, and support must be adequate; injury to the sites of recent corrective surgery must be guarded against.

\section{Multiple surgical procedures}

Many of these corrective orthopaedic procedures may be performed simultaneously. As many as three tourniquets may be applied at one time. This is contraindicated in the rheumatoid patient with precarious cardiovascular and haemopoietic haemodynamics, since central venous filling (CVP) and cardiac output (co) will be significantly altered by this technique. Tourniquet release may also be associated with a metabolic acidosis, as a result of release into the systemic circulation of acid metabolites that have accumulated in the occluded limb. Significant metabolic acidosis, may impair muocardial contractility. Intravenous sodium bicarbonate can be given prophylactically and/or therapeutically to combat this effect.

\section{Postoperative Period}

\section{Respiratory hazards}

Because of the airway problems in these patients extubation is deferred until there is ample evidence that the patient is able to maintain an airway and that 
spontaneous ventilatory capacity is adequate. This is usually clearly indicated when the tidal volume, minute volume, and arterial blood gases $\left(\mathrm{Po}_{2}, \mathrm{Pco}_{2,}, \mathrm{pH}\right)$ are stable and within normal limits and the patient is conscious enough to remove the endotracheal tube himself. Patients with marked fixation and deformity of the thoracic spine and costovertebral involvement may require assisted ventilation with IPPB for several hours postoperatively. This management is not necessarily prolonged as the anaesthetic is usually designed to provide early awakening and minimal postanaesthetic depression. However, because multiple joint surgery may have been performed, these patients may not be active in the early postoperative period and may be prone to develop atelectasis. Follow-up chest physiotherapy is essential.

Care should be exercised in the use of potent respiratory depressing narcotic analgesics in the postoperative period. Deaths in rheumatoid patients have resulted from drug-induced respiratory failure in this period. ${ }^{3}$

\section{Fluid and electrolyte hazards}

Rheumatoid patients are often slight of build, and frequently adults may weigh only $35 \mathrm{~kg}$ or less. Routine adult fluid balance orders may precipitate a dilutional hyponatremia and water intoxication with overt convulsive manifestations.

Rheumatoid arthritis is a crippling, incapacitating disease. Corrective surgical procedures offer new hope and great potentiality for its victims. Care in their anaesthetic management can significantly contribute to the successful outcome of these procedures.

\section{SUMMARY}

Rheumatoid arthritis is a relatively common disease for which surgical treatment is being employed with increasing frequency. The improved results are the product of the co-ordinated efforts of many medical and paramedical disciplines. The skill of anaesthetic management is an important contributing factor.

From our experiences during the past five years, special problems can be anticipated. These are: airway impairment (secondary to hypoplastic mandible, temporomandibular joint ankylosis, cervical spine restriction, atlanto-axial subluxation, low cervical subluxation, cricoarytenoid arthritis and laryngeal tissue damage); respiratory inadequacy (rheumatoid lung nodules, chronic lung diffusing interstitial fibrosis, costovertebral joint fixation, and thoracic vertebral fixation deformity); cardiovascular impairment (pericardium, myocardium, coronary artery, regurgitant aortic valve involvement, and disseminated necrotizing arteritis); haemopoictic defects (anaemia, leucopenia, and bleeding tendencies); renal and hepatic dysfunction; drug therapy hazards; problems in posturings; and hazards associated with multiple anaesthetics and surgical procedures.

Advanced rheumatoid arthritis is a crippling, incapacitating disease. Corrective surgical procedures offer new hope and great potentiality for its victims. Care in the anaesthetic management of these patients can significantly contribute to the successful outcome of these procedures. 


\section{REFERENCES}

1. Harrison, T. R. (Ed). Principles of Internal Medicine. 5th edition. New York. McGrawHill Book Co. Inc. (1966), p. 1352.

2. Edellst, G. Principles of Anaesthetic Management in Rheumatoid Arthritic Patients. Anesthesia and Analg. Curr. Res. 43: 227-231 (1964).

3. Gardnen, D. L. \& Holmes, F. Anaesthetic and Post-operative Hazards in Rheumatoid Arthritis. Brit. J. Anaesth. 33: 258 (1961).

4. Brenenstock, H.; Ehrlich, E.; \& Grexberg, R. H. Rheumatoid Arthritis of the Gricoarytenoid Joint: A Clinico-pathological Study. Arthritis Rheum. 6: 48 (1963).

5. Sieniewicz, D. J.; Martin, J.; Moore, S.; \& Mittara, M. Rheumatoid Nodules in the Lung. J. Assoc. Radiol. 13: 73 ( 1962 ).

6. Caplan, A. Certain Unusual Radiological Appearances in the Chest of Coal Miners Suffering from Rheumatoid Arthritis. Thorax. 8: 29 (1953).

7. Lee, F. I. \& Brain, A. T. Chronic Diffuse Interstitial Pulmonary Fibrosis and Rheumatoid Arthritis. Lancet 2: 693 (1962).

8. Catricart, E. S. \& Spodick, D. H. Rheumatic Heart Disease: A Study of the Incidence and Nature of Cardiac Lesions in Rheumatoid Arthritis. New Eng. J. Med. 266; 959 (1962).

9. Gramam, D. C. \& SMythe, H. N. The Carditis and Aortitis of Ankylosing Spondylitis. Bull, Rheum. Dis. 9: 171 (1958).

10. Rushmer, R. F. Cardiovascular Dynamics. 2nd edition. Philadelphia. W. B. Saunders Co. (1961).

11. Ferguson, R. H. \& Slocumb, C. H. Peripheral Neuropathy in Rheumatoid Arthritis. Bull. Rheum. Dis. 11: 251 (1961).

12. McGraw, R. Personal Communications (1968).

13. Nunn, J. F. \& Freeman, J. Problems of Oxygenation and Oxygen Transport in Anaesthesia. Anaesthesia 19: 120 (1964).

14. Dal Santo, G. Kinetics of Distribution of Radioactive Labeled Muscle Relaxants: III. Investigations with ${ }^{14} \mathrm{C}$-succinyldicholine and ${ }^{14} \mathrm{C}$-succinylmonocholine During Controlled Conditions. Anesthesiology 29: 435-443 (1968).

15. Sorenson, A. W. S. Investigations of the Kidney Function in Rheumatoid Arthritis. Acta. Rheum. Scand. 7: 138 (1961).

16. JACKes, A. M. Renal Amyloid: A report on eleven cases proven by renal biopsy. Ann. Rheum. Dis. 19: 126 (1960).

17. Nettlenladt, E. On Some Liver Functions in Rheumatoid Arthritis. Acta. Rheum. Scand. 8: 63 (1962).

18. Van Dyke, R. A. \& Chenoweth, M. B. Review: Metabolism of Volatile Anesthetics. Anesthesiology. 26: $348-357$ (1965).

19. Featherstone, R. M; Muehllbancher, C. A.; De Bon, F. L.; \& Forsaith, J. A. Interactions of Inert Anesthetic Gases with Proteins. Anesthesiology. 22: 977 (1961).

20. Martel, W. \& PAge, J. W. Cervical Vertebral Erosions and Subluxations in Rheumatoid Arthritis and Ankylosing Spondylitis. Arthritis Rheum. 3: 546 (1960). 